\title{
Antropocentrismo y ecocentrismo en la jurisprudencia de la Corte Interamericana de Derechos Humanos ${ }^{1}$
}

\section{Anthropocentrism and Ecocentrism in the Jurisprudence of the Inter-American Court of Human Rights}

\author{
Digno Montalván Zambrano ${ }^{2}$ \\ Universidad Carlos III de Madrid (España) \\ ORCID: https://orcid.org/0000-0002-6050-1777
}

Recibido: 17-01-2020

Aceptado: 07-06-2020

\section{Resumen}

El artículo analiza la presencia del argumento antropocéntrico y ecocéntrico en la jurisprudencia de la Corte Interamericana de Derechos Humanos relacionada con temas ambientales. A tal efecto, en un primer momento, presenta el contenido de estas dos aproximaciones dando cuenta de sus aportes y limitaciones. Con este marco teórico, en un segundo momento, identifica cuatro etapas de la jurisprudencia de la Corte, las cuales muestran el tránsito paulatino del antropocentrismo al ecocentrismo dentro de sus reflexiones sobre los derechos humanos. Finalmente, los resultados del análisis ofrecen luces sobre el posible desarrollo del derecho al ambiente sano como derecho autónomo en el Sistema Interamericano de Derechos Humanos.

\footnotetext{
${ }^{1}$ La realización de este trabajo ha tenido lugar en el marco del proyecto de investigación "Jueces en Democracia. La filosofía política de la Corte Interamericana de Derechos Humanos" (DER201679805-P, AEI/FEDER, UE), y del proyecto "Teorías de la justicia y derecho global de los derechos humanos" (PID2019-107172RB-I00), ambos financiados por el Ministerio de Ciencia e Innovación de España y adscritos al Grupo de Investigación sobre el Derecho y la Justicia de la UC3M.

2 (dmontalv@clio.uc3m.es) Co-coordinador del taller sobre el derecho y la justicia de la UC3M y editor de la Revista Eunomía. Sus publicaciones más recientes son: "El pluralismo jurídico y la interpretación intercultural en la jurisprudencia constitucional de Ecuador y Bolivia" (Ratio Juris, 2019); "Justicia ecológica" (Eunomía, 2020); "El derecho al medio ambiente sano como derecho autónomo en el SIDH” (Anales de la Facultad de Derecho, 2020).
} 
Palabras-clave: Corte Interamericana de Derechos Humanos, ecocentrismo, antropocentrismo, derecho al ambiente sano, derechos de la naturaleza.

\begin{abstract}
The article analyzes the presence of the anthropocentric and ecocentric argument in the jurisprudence of the Inter-American Court of Human Rights related to environmental issues. To this effect, at first, it presents the content of these two approaches, explaining their contributions and limitations. With this theoretical framework, identifies four stages in the Court's jurisprudence, which show the gradual transition from anthropocentrism to ecocentrism within its reflections on human rights. Finally, the results of the analysis offer light on the possible development of the right to a healthy environment as an autonomous right in inter-American human rights system.
\end{abstract}

Key-words: Inter-American Court of Human Rights, Ecocentrism, Anthropocentrism, Right to a Healthy Environment, Rights of Nature.

\title{
1. Introducción
}

"Para los efectos de esta Convención, persona es todo ser humano". De esta forma define la Convención Americana de Derechos Humanos (en adelante "la Convención"), en su artículo 1.2, al sujeto de protección dentro del sistema interamericano. Con ello, conforme lo expresó el ex juez de la Corte Interamericana de Derechos Humanos (en adelante "Corte IDH"), Sergio García Ramírez, se reproduce, en el texto y espíritu de la Convención, la proclama del constitucionalismo antropocéntrico de los siglos XVIII, XIX y XX de que la norma, como dogma de humanismo, "acredita la convicción de que la sociedad política fue instituida para la protección de los derechos naturales y la felicidad del pueblo"3. Bajo esta aproximación, los intereses del ser humano serían el centro del discurso moral que predica la Convención, guardando, con ello, fidelidad al fundamento que inspira su origen, la razón antropocéntrica kantiana. Este artículo no rechaza la afirmación anterior ${ }^{4}$, por el contrario, partiendo de su indispensable existencia, propone dar cuenta de cómo, desde este paradigma, un otro, la naturaleza, ha sido excluido del discurso de los derechos, dando paso a interpretaciones que, en favor del supuesto cumplimiento de fines humanos, han legitimado tanto su depredación indiscriminada como su instrumentalización.

\footnotetext{
3 García Ramírez, S. Voto razonado dentro de la sentencia del caso Comunidad Indígena Sawhoyamaxa Vs. Paraguay, de 29 de marzo de 2006, párr. 18.

${ }^{4}$ Respecto de los aportes del concepto de dignidad humana kantiano en la fundamentación de los derechos humanos y, en específico, sobre su presencia en la Corte IDH, se puede consultar: (Delgado, 2018 y 2020).
} 
Para el antropocentrismo kantiano, el humano, único ser racional dentro del mundo natural, es el sujeto y objeto exclusivo de la moralidad, el portador único de dignidad y, por ende, de su traducción jurídica: el derecho a tener derechos. Frente a esto, el ecocentrismo, partiendo de una visión holística de lo humano, propone ampliar nuestras reflexiones sobre la comunidad moral, cuestionando la idea antropocéntrica del daño y, con ello, nuestra exclusividad como sujetos de derechos. Las Constituciones de Ecuador de 2008 y Bolivia de 2009, así como los avances jurisprudenciales en altas cortes de diferentes países de América, dan cuenta de cómo, dentro del continente, dicho cuestionamiento adquiere una creciente relevancia dentro del discurso jurídico. Este cuestionamiento, a su vez, parece impactar en el Sistema Interamericano por medio del recientemente consagrado derecho al medio ambiente sano como derecho autónomo. Este derecho, conforme lo señala la Corte IDH, busca proteger a la naturaleza como un bien en sí mismo, aun en ausencia de riesgo para el ser humano. Concentra el daño en la naturaleza y cuestiona con ello la consideración del ser humano como el único sujeto de protección al que se referiría el artículo 1.2 de la Convención. Se aproxima a una visión ecocéntrica, la cual, si bien ofrece importantes ventajas para la protección de los sujetos no humanos, al mismo tiempo abre grandes interrogantes: ¿es coherente hablar de un enfoque ecocéntrico de los derechos humanos? y de ser afirmativa la respuesta, ¿por qué hacerlo?

A estas preguntas pretende responder el primer apartado de este trabajo. Se consideran elementos previos indispensables para el estudio casuístico posterior, pues si nada tuviera que decir el ecocentrismo al interior de los derechos humanos, poco sentido tendría rastrear su presencia. Con ello, la segunda parte del artículo presenta la evolución del lugar de la naturaleza en la Corte IDH. Su objetivo es dar cuenta de que el giro ecocéntrico que planteo no es un anecdótico ni sorpresivo quiebre jurisprudencial; por el contrario, su aparición parece ser resultado de un proceso de evolución jurisprudencial paulatino, el cual ha acompañado la creciente relevancia del tema ambiental en el debate filosófico, social y político. Así, se identifican cuatro momentos en la jurisprudencia de la Corte IDH sobre temas ambientales, los cuales, de la mano con el objetivo de este trabajo, explican la influencia del argumento antropocéntrico y ecocéntrico en la evolución del debate sobre los derechos humanos, sus aportes, limitaciones y contradicciones.

\section{2. ¿Es posible un giro ecocéntrico en la jurisprudencia de la Corte IDH?}

A primera vista puede resultarnos una contradicción insalvable el que un no humano sea sujeto de protección dentro de un sistema que, en su origen, 
se fundamenta en la exclusiva preservación de los intereses/derechos de los seres humanos. Se puede considerar más acertado, por ejemplo, la creación de un nuevo sistema internacional de protección dirigido de forma exclusiva a la naturaleza; entonces, ¿por qué plantear la incorporación de un enfoque ecocéntrico en los derechos humanos?

En mi opinión, dicha incorporación resulta no solo relevante, sino, además, necesaria por dos motivos: uno estratégico y otro sustantivo. Respecto del primero, concentrar en un único sistema los derechos de la naturaleza y los derechos de los seres humanos permite evitar los conflictos habituales entre jurisdicciones internacionales. Estos derechos, vistos como distintos, tienden a superponerse y a disputar sus espacios frente al otro. Así, enfrentarlos dentro de un mismo tribunal permite un diálogo más fructífero y una mirada más holística respecto de los bienes jurídicos en juego. En segundo lugar, como se dijo, la fundamentación tradicional de los derechos humanos ha partido de una mirada liberal-antropocéntrica, pero esto no ha sido ni tiene por qué ser así. Nuevas demandas sociales vienen impulsando otras posibles fundamentaciones de los derechos humanos, en algunos casos incorporando correcciones al modelo liberal y, en otros, superándolo por completo. Pero llevar derechos más allá de los límites que su antecedente filosófico propone genera tensiones que, de no ser evidenciadas, pueden amenazar espacios que ya se creían conquistados, incluso frente liberalismo. En el caso del ecologismo, su creciente influencia ha dado paso a interpretaciones que, como veremos, disputan espacios al derecho a la propiedad comunal de los pueblos indígenas, el cual, en sí mismo, ya constituía una conquista frente al liberalismo. En este marco, y por razones que expondré a continuación, considero no solo factible sino, además, necesaria la incorporación de un enfoque ecocéntrico en la fundamentación de los derechos humanos.

Bajo el ecocentrismo la naturaleza tiene una visión dialéctica, esto es, a diferencia de su tratamiento instrumental-antropocéntrico pasa a tener papeles activos y pasivos en la relación con el ser humano (Costa, 2009: 115). Su enfoque es teleológico, se centra en los ciclos vitales de la naturaleza y apuesta por una justicia ecológica que mira hacia los procesos biogeoquímicos y físicos que crean las condiciones que permiten la vida sobre la tierra y de los cuales el ser humano es parte (Klett \& Martínez, 2013: 11,12). Los derechos de la naturaleza no olvidan, entonces, que las dinámicas ecológicas implican también relaciones de competencia y depredación en las que se incluye el humano (Gudynas, 2011: 272). Estas características hacen que, en mi consideración, este sea el único enfoque coherente para una posible incorporación de la naturaleza como sujeto de derechos en el discurso de los derechos humanos, pues: 1) no discute la existencia de intereses antropocéntricos, es más, los incorpora como parte de las relaciones 
que se dan en el mundo natural y, de la mano con lo anterior, 2) forma parte de su esencia la tensión entre el ser humano y el resto de la naturaleza. De este modo, el ecocentrismo, sin desconocer al antropocentrismo, permite abrir las puertas a otro tipo de discusión en el derecho, la política y la gestión, donde, en espacios de colisión entre intereses humanos y derechos la naturaleza, ya no sea necesario "demostrar que preservar montañas o selvas es útil para el ser humano, o es rentable para las empresas, sino que las fundamentaciones por su valoración intrínseca serán tan importantes como los análisis costo beneficio de los economistas" (Gudynas, 2010: 53).

En suma, se propone redefinir aquello que entendemos por lo "humano". Superar la visión de sujeto "racional independiente", ajeno a la naturaleza, y construir una nueva categorización que responda a la interrelación y cooperación con el mundo natural que nos permiten Ser y definirnos. Así, lo humano no es únicamente lo antropocéntrico; este, tal como lo establece la Corte Suprema de Colombia, se construye también a partir del respeto a la parte de sí mismo que está compuesta por la naturaleza 5 . Dentro de esta nueva posible fundamentación de los derechos humanos, la naturaleza es un bien en sí mismo porque es un "otro" con el cual me defino. No es ajena a mí, me constituye y construye desde su otredad. El respeto por sus intereses, por sus fines, es, por tanto, el respeto por la parte de mí, ser humano, que se construye en el viaje conjunto con esos otros seres sintientes o no, que posibilitan las redes de vida en la que nos encontramos insertos.

Esta nueva interpretación de "lo humano", claramente ecocéntrica, nos permite incorporar en la lectura del artículo 1.2 de la Convención a la naturaleza como sujeto de protección. Sin embargo, con ello no desterramos la visión antropocéntrica presente en la Convención; por el contrario, como se dijo, su existencia se considera un punto de partida indispensable. Esto hace posible escenarios en los que estas dos aproximaciones al Ser puedan verse enfrentadas; sin embargo, en mi consideración, la tensión que nace de dicho encuentro no es irresoluble ni necesariamente dañina. Tal como se refleja entre otros derechos humanos, la tensión presente entre bienes jurídicos puede producir, en su colisión práctica, el fortalecimiento y precisión de los derechos en juego.

Desde esta línea, esto es, considerando posible la incorporación de un enfoque ecocéntrico en el Sistema Interamericano y mirando la tensión entre los derechos humanos y derechos de la naturaleza como una oportunidad para la armonización y ampliación de nuestra definición como sujetos "humanos", parte el presente trabajo.

${ }^{5}$ Corte Suprema de Colombia (2018). Sentencia 4360-2018 de 5 de abril de 2018, p. 21.

Araucaria. Revista Iberoamericana de Filosofía, Política, Humanidades y Relaciones Internacionales, año $23, \mathrm{n}^{\circ} 46$. Primer cuatrimestre de 2021. Pp. 505-527. ISSN 1575-6823 e-ISSN 2340-2199 https://dx.doi.org/10.12795/araucaria.2021.i46.25 


\section{Análisis jurisprudencial: antropocentrismo y ecocentrismo en la Corte IDH}

En los próximos apartados me dedicaré a presentar cómo han impactado el antropocentrismo y el ecocentrismo en la jurisprudencia de la Corte IDH. Para lo anterior, tomo como eje el valor que ha tenido la naturaleza dentro de su interpretación de los derechos humanos. Producto de este análisis, se han identificado cuatro momentos de su jurisprudencia, los cuales detallaré a continuación.

\subsection{Su completa instrumentalización}

a diferencia de las bestias o de los objetos inanimados, el hombre posee atributos sobrenaturales o divinos que lo hacen acreedor al respeto de sus semejantes (Trejos Fernández, 1978: 407, 408).

Con estas palabras daba inicio el presidente de Costa Rica a la conferencia producto de la cual, el 22 de noviembre de 1969, se definiría el actual texto de la Convención. La esencia del postulado antropocéntrico aparece aquí de forma contundente, la visión del ser humano como distinto y superior a la naturaleza, aquello que Kant denominó el "fin último de la creación" (1876 [1790]: 119). Expresa la consideración cartesiana de lo natural como "cosa" a disposición ilimitada del ser humano (Descartes, 2002 [1637]: 14). Bajo esta postura, el humano, en tanto es medida, origen y destino de todos los valores, se apropia de los recursos naturales al entenderlos únicamente como "medios" a su disposición.

En esta primera etapa la naturaleza no existe. No hay rastros de ella en los proyectos ni en el texto definitivo de la Convención. Es más, durante los primeros 22 años de existencia de la Corte, las palabras ambiente o naturaleza no aparecen en su jurisprudencia, su preservación no era parte de los derechos humanos. Muy por el contrario, si revisamos el artículo 25 del primer proyecto de Convención veremos que, siguiendo el artículo 34 de la Carta de la OEA, se establecen la modernización de la vida rural, la mayor productividad agrícola y la expansión del uso de la tierra como parte de los derechos económicos, sociales y culturales. Este proyecto de artículo, cuyo texto fue reemplazado por el del actual artículo 26 de la Convención, marca la primera etapa de la relación entre los derechos humanos y la naturaleza en el sistema interamericano, esto es, la del derecho al desarrollo como dominación indiscriminada del medio ambiente. 


\subsection{La protección indirecta de tipo antropocéntrica}

Esta segunda etapa se inaugura con el caso Awas Tingni Vs. Nicaragua, de $2001^{6}$ y se extiende hasta el caso Saramaka Vs. Surinam de 20077. Tiene como fuente el liberalismo kantiano, el cual admite obligaciones humanas hacia el ambiente como resultado de la propia dignidad humana, es decir, admite el hecho de que el ser humano se desenvuelve dentro de un "ambiente" al cual transforma y, aunque externo él, lo condiciona (Kant, 1988 [1775-1781]: 290). El modelo de justicia derivado de esta aproximación es la justicia ambiental; para esta lo justo o injusto se determina a partir de los efectos que para las personas puede tener el daño al ambiente. Su traducción en el mundo de los derechos humanos es el derecho a un ambiente sano. Este derecho se fundamenta en la necesidad de la preservación del ambiente para el disfrute de otros derechos humanos, tales como el derecho a la vida o a la integridad física. Se enmarca, por tanto, en lo que varios autores denominan un enfoque instrumental de la protección del medio ambiente para la garantía de los derechos humanos y, en la vertiente inversa, un enfoque instrumental de los derechos humanos para la protección del medio ambiente (Shelton, 2010: 111-114).

El caso Awas Tingni Vs. Nicaragua marca el inicio de esta etapa. Aquí se demandó la vulneración del derecho a la propiedad por la construcción de carreteras y explotación de madera en tierras de la comunidad Awas Tingni. Este constituye el primer caso en el que la Corte reconoce el derecho a la propiedad comunal de los miembros de pueblos indígenas, con base en el estrecho vínculo que mantienen con su tierra (párr. 149). De esta forma, incorpora por primera vez la relación ser humano-naturaleza en su reflexión sobre los derechos humanos y, por esta vía, la importancia de la conservación del ambiente para las generaciones futuras.

Así lo expresaron los jueces Cançado, Pacheco y Abreu en su voto razonado conjunto, al dar cuenta de la importancia que, para el fallo, tuvo la concepción del hábitat de la comunidad Awas Tingni ${ }^{8}$. El hábitat, como resaltan los citados jueces, adquiere para estos una dimensión intertemporal (párrs. 6-9), es decir:

se proyecta tanto en el espacio como en el tiempo, por cuanto nos relacionamos, en el espacio, con el sistema natural de que somos parte y que debemos tratar con cuidado, y, en el tiempo, con otras generaciones (las pasadas y las futuras), en relación con las cuales tenemos obligaciones (párr.10).

\footnotetext{
${ }^{6}$ Corte IDH. Sentencia del caso de la Comunidad Mayagna (Sumo) Awas Tingni Vs. Nicaragua, de 31 de agosto de 2001.

${ }^{7}$ Corte IDH. Sentencia del caso del Pueblo Saramaka vs. Surinam, de 28 de noviembre de 2007.

${ }^{8}$ Cançado, A; Pacheco, M., y Abreu, A. (2001). Voto razonado conjunto, caso Comunidad Mayagna (Sumo) Awas Tingni Vs. Nicaragua, de 31 de agosto de 2001.
}

Araucaria. Revista Iberoamericana de Filosofía, Política, Humanidades y Relaciones Internacionales, año $23, \mathrm{n}^{\circ} 46$. Primer cuatrimestre de 2021. Pp. 505-527. ISSN 1575-6823 e-ISSN 2340-2199 https://dx.doi.org/10.12795/araucaria.2021.i46.25 
La idea de que nos relacionamos en el espacio con el sistema natural del que somos parte refleja elementos de la visión ecocéntrica propia de la, en principio, armónica relación que los pueblos indígenas mantienen con su tierra. Es importarte resaltar esto, pues da cuenta de cómo, en la primera de las sentencias sobre cuestiones ambientales de la Corte, ya se encontraba presente un enfoque ecocéntrico en los argumentos dados por las partes; sin embargo, como veremos, este es traducido a términos antropocéntricos por los jueces. En el caso, la relación de los miembros de la comunidad indígena con su tierra es considerada por los citados jueces como una manifestación cultural que forma el substratum de las normas jurídicas que deben regir las relaciones de los comuneros inter se y con sus bienes (párr. 11). Es decir, son las relaciones culturales específicas de este pueblo las que, llevadas al análisis de las afectaciones del caso, legitiman la interpretación evolutiva del derecho a la propiedad. Así, se debe conservar la naturaleza porque en ella se desarrolla un elemento cultural humano que se debe preservar, no porque importe en sí misma. El carácter instrumental es claro y, en el caso, lleva a que no se contemple ninguna medida de reparación destinada a la reforestación de las áreas afectadas.

Del mismo modo, la idea de que en el tiempo la relación ser humanonaturaleza se expresa con otras generaciones (pasadas y futuras), responde a una visión antropocéntrica. Aquí, una vez más, la naturaleza carece de valor propio, su cuidado es importante en tanto garantiza la vida para las generaciones humanas venideras. Al respecto, y sin pretender desmerecer los aportes que para la preservación del ambiente ofrece esta perspectiva, dentro del Sistema Interamericano, por razones que veremos a continuación, la fundamentación de los derechos humanos a partir de esta idea parece más una expresión retórica que una auténtica ventana de oportunidad para la protección de la naturaleza.

Volviendo al artículo 1.2 de la Convención, su interpretación, en línea con el liberalismo antropocéntrico que inspira este momento de la jurisprudencia de la Corte, daba cuenta de que ahí donde se leía "persona es todo ser humano", se estaba refiriendo, de forma exclusiva, a "los derechos del individuo, del ser humano de carne y hueso" (Gros, 1991: 72). Dos conclusiones podemos sacar de esta primera aproximación: 1) solo "el individuo", es decir, la persona singular, podía ser considerada víctima. Bajo esta consideración, por ejemplo, la Corte negó hasta el 2012 toda posibilidad de reconocimiento del carácter de víctima a comunidades indígenas en tanto tales ${ }^{9}$, y 2) esa persona debía de ser de "carne y hueso", es decir, debe ser un sujeto humano presente. Esta segunda conclusión resulta central al momento de determinar qué enfoque es más adecuado para la protección de la naturaleza en el sistema interamericano. Bajo esta interpretación, por ejemplo, la Corte IDH, en el caso Gómez Murillo y

${ }^{9}$ Un estudio interesante sobre el tema lo podemos encontrar en: (Wences y Russo, 2016) 
otros VS. Costa Rica ${ }^{10}$, sobre fecundación in vitro, determinó que el embrión no podía ser considerado persona y, por ende, tampoco sujeto de protección (párr. 264). El punto clave de esta interpretación, llevada al asunto del que se ocupa el presente artículo, es que niega la protección a "seres" que no existen aún (párrs. 176-190; 221-223; 244). Así, la propuesta antropocéntrica de protección del ambiente para las generaciones humanas futuras ${ }^{11}$ (es decir, para "seres" que no existen aún), fundamentada en la idea de justicia intergeneracional, no parece posible dentro del Sistema Interamericano.

Puntualizado lo anterior, y avanzando con el análisis jurisprudencial, el siguiente gran paso de la Corte en la protección del ambiente desde la vertiente antropocéntrica lo encontramos en la sentencia del caso comunidad indigena Yakye Axa Vs. Paraguay, de 2005 ${ }^{12}$. Los hechos se relacionan con el desplazamiento sufrido por los miembros de esta comunidad, por la venta de sus tierras a través de la bolsa de valores de Londres y posterior instalación de estancias ganaderas en la zona (párrs. 29-30). En este caso, la Corte vincula, por primera vez, el derecho al ambiente sano (art. 11 del protocolo de San Salvador) con el derecho a la vida, aunque no desarrolla nada al respecto (párr. 163). En esta misma línea, a pesar de que la Comisión había solicitado que se exigiera al Estado proteger las tierras reclamadas a fin de que no fueran extraídos sus recursos naturales, en especial sus bosques, la Corte no dispuso ninguna medida de reparación en ese sentido (párr. 207).

Por otro lado, el 19 de septiembre de 2006, la Corte IDH emite la sentencia del caso Claude Reyes y otros Vs. Chile ${ }^{13}$. Este versa sobre la vulneración del derecho al acceso a la información pública por la negativa del Estado de brindar información relativa a un proyecto de deforestación. El precedente relevante es la categorización de la información relacionada con actividades y proyectos que puedan tener impacto ambiental como asunto de interés público. Producto de lo anterior, conforme lo establece la Corte dicha información debe ser entregada sin necesidad de acreditar un interés directo para su obtención o una afectación personal (párrs. 73-77). La importancia de este precedente es, sin duda, enorme; la garantía del acceso a la información pública en estos términos permite un mayor escrutinio sobre temas ambientales. Sin embargo, se debe precisar que, desde la aproximación antropocéntrica que marca esta etapa, el recurso a la defensa del derecho al acceso a la información no expresa, en su interior, un interés real por la preservación de la naturaleza. Así lo prueba el no pronunciamiento de la Corte respecto de la solicitud del

\footnotetext{
${ }^{10}$ Corte IDH (2016). Sentencia del caso Gómez Murillo y otros Vs. Costa Rica, de 29 de noviembre de 2016.

${ }^{11}$ Algunos autores que proponen un modelo de justicia intergeneracional antropocéntrica son: (Jonas, 2015); (Dobson, 2005) y (Máiz, 2011).

${ }^{12}$ Corte IDH (2005). Sentencia del caso Comunidad indigena Yakye Axa Vs. Paraguay, de 17 de junio de 2005.

${ }^{13}$ Corte IDH. Sentencia del caso Claude Reyes y otros Vs. Chile, de 19 de septiembre de 2006.
} 
demandante de que, como medida de reparación, el Estado de Chile genere de forma oficiosa información en materia ambiental (transparencia activa) (párr. 146). Este silencio, en mi consideración, da cuenta de que en este caso la cuestión ambiental aparece de forma meramente accidental. Lo único que la Corte tiene como elemento de reflexión es el derecho humano al acceso a la información vinculado a la libertad de expresión, es decir, a recibir respuestas de las autoridades públicas (transparencia pasiva).

Finalmente, el último caso de esta etapa y, tal vez, el más claro ejemplo de los límites de la protección antropocéntrica del ambiente, lo constituye la sentencia del caso del pueblo Saramaka Vs. Surinam de 200714. Este versa sobre el otorgamiento de concesiones estatales para actividades de explotación forestal y minera, que habrían causado daño al ambiente (párr. 124). El problema jurídico, conforme se plantea en la sentencia, gira alrededor de quién posee la titularidad de los recursos naturales. La respuesta de la Corte al respecto inicia una línea jurisprudencial que, como veremos en la última de las etapas, parece estar cambiando. Me refiero a la intrínseca e inseparable relación entre el derecho a la propiedad y el derecho al territorio de los pueblos indígenas (Wences y Russo, 2016: 289).

Así lo expresó la Corte IDH en el párrafo 122 de esta sentencia, al señalar que, "debido a la conexión intrínseca que los integrantes de los pueblos indígenas y tribales tienen con su territorio, es necesaria la protección del derecho a la propiedad sobre éste, de conformidad con el artículo 21 de la Convención, para garantizar su supervivencia". El efecto de lo anterior en materia de protección jurisdiccional es vincular, de forma inseparable, toda violación del derecho al territorio (al acceso a los recursos naturales) con una violación al derecho de propiedad (a la titularidad de la tierra en la que habitan) (párr. 122). La conclusión que quiero señalar por el momento es que esta inseparable vinculación entre recursos naturales y derecho a la tierra expresa dos consideraciones: 1) aquí la protección de la naturaleza importa en tanto que recurso indispensable para la supervivencia de las comunidades indígenas, y, como consecuencia de aquello, 2) el concepto de territorio se limita al espacio físico en el que habitan y del que son propietarios. Este último punto, como veremos, será replanteado en la etapa que clasifico como del giro ecocéntrico, no exento de cuestionamientos.

Sobre la primera consideración, el punto de partida antropocéntrico del mismo lleva a la Corte a proteger de forma exclusiva los intereses de ese grupo de personas humanas. Así lo refleja en el párrafo 126 de la sentencia, al indicar que el agua y los bosques son esenciales en tanto recursos naturales en los que el pueblo Saramaka realiza algunas de sus actividades económicas de subsistencia, como la caza y la pesca. Los argumentos relativos a la

\footnotetext{
${ }^{14}$ Corte IDH. Sentencia del caso del Pueblo Saramaka vs. Surinam, de 28 de noviembre de 2007.
} 
preservación de la naturaleza son, así, meramente instrumentales. Esto lleva a que otro de los grandes pasos que da la Corte con esta sentencia, esto es, hacer referencia a la idea de daño ambiental (párr. 154), carezca de efecto práctico en materia de conservación. De esta forma, a pesar de que la Comisión había solicitado como reparación la recuperación del daño ambiental causado (párr. 191), la Corte no dicta ninguna medida al respecto. Al referirse al daño material se enfoca de forma exclusiva en las repercusiones económicas que para el pueblo Saramaka tuvo la extracción de una "cantidad considerable de madera valiosa (...) sin antes consultarle o brindarle una indemnización" (párr. 191). La sentencia se encuadra, así, en un modelo antropocéntrico de justicia distributiva ecológicamente corregida (Gudynas, 2010: 59). Esta, como lo ha expresado Leff, resulta obsoleta para dar cuenta de las estructuras ecológicas y tecnológicas que determinan las condiciones que hacen que mejore o se degrade el estado de conservación y la productividad de un ecosistema (1995: 29-30). Las indemnizaciones económicas para los humanos no implican, en sí mismas, medidas que favorezcan la naturaleza.

Con este antecedente, la pregunta que quiero abrir para concluir este apartado es ¿cuál es el lugar real de la naturaleza en este enfoque? ¿Hay oportunidades reales para su cuidado? Imaginemos un escenario en el que el modelo de justicia distributiva antropocéntrica, expresada a modo de participación en los beneficios, se aplique de forma incuestionable. Las cargas y compensaciones ambientales se reparten de forma equitativa en la sociedad. En este caso hipotético, además, la comunidad indígena ve mejorada considerablemente su calidad de vida, es más, se integra a la capitalización de los beneficios de la actividad extractiva. En este escenario, el antropocentrismo difícilmente cuestionaría algo. Sin embargo, a lo largo del ejemplo, jamás ha adquirido un rol protagónico la naturaleza. Su instrumentalización, en este caso, no le ha llevado a su preservación, todo lo contrario, el fin último del reino de los fines ha sentenciado su destino: servir a la mejora del ser humano.

\subsection{El inicio de la tensión ambiente-derechos humanos}

Esta etapa, a la que considero de transición, se inaugura con la sentencia del caso Salvador Chiriboga Vs. Ecuador de $2008^{15}$ y se extiende hasta el caso de los pueblos Kaliña y Lokono Vs. Surinam, de noviembre de $2015^{16}$. Aquí la Corte, aun sin reconocer a la naturaleza como poseedora de intereses propios, le otorga un peso sustancial al momento de reflexionar sobre el alcance de

\footnotetext{
${ }^{15}$ Corte IDH. Sentencia del caso Salvador Chiriboga Vs. Ecuador, de 6 de mayo de 2008.

${ }^{16}$ Corte IDH. Sentencia del caso pueblos Kaliña y Lokono vs. Surinam de 25 de noviembre de 2015 .
}

Araucaria. Revista Iberoamericana de Filosofia, Política, Humanidades y Relaciones Internacionales, año $23, \mathrm{n}^{\circ} 46$. Primer cuatrimestre de 2021. Pp. 505-527. ISSN 1575-6823 e-ISSN 2340-2199 https://dx.doi.org/10.12795/araucaria.2021.i46.25 
los derechos establecidos en la Convención. Este peso, sin embargo, no es explícito. Son frases dispersas entre el texto de estas sentencias, cuya relevancia podría parecer menor, las que comienzan, a mi juicio, a dar cuenta de cómo la naturaleza, de a poco, va tomando un papel protagónico al interior de la Corte.

La poca visibilidad dada al real peso que adquiere el discurso ambiental en esta etapa, produce, a su vez, tensiones ocultas en sentencias que, en principio, parecerían no alejarse del marco antropocéntrico de reflexión. Esta tensión la identifico con el reconocimiento de la protección de la naturaleza como interés jurídico relevante para la restricción de derechos. A partir de aquello, se incorpora a la naturaleza no solo como condición para la realización de los derechos del ser humano (relación sin tensión), sino, además, como argumento para su limitación (relación con tensión).

Esta etapa inicia con el caso Salvador Chiriboga Vs. Ecuador, en el cual se demandó la declaración de utilidad pública y correspondiente expropiación de un predio privado para la creación un área de recreación y protección ecológica (párr. 71). En este, la Corte reconoce, por primera vez, que un interés social basado en la protección del medio ambiente representa una causa de utilidad pública legítima para la privación del derecho de propiedad privada (párr. 76). De esta manera, en apenas dos frases, la Corte incluye el ambiente como un elemento a tener en cuenta, ya no solo para la protección de los derechos humanos, sino, además, para su posible limitación. Dos años más tarde, en la sentencia del caso comunidad indígena Xákmok Kásek VS. Paraguay ${ }^{17}$, la primera sobre reservas naturales (en este caso privadas) y derechos de pueblos indígenas, la Corte llama la atención sobre la utilización, precisamente, del discurso ambiental para la restricción injustificada de derechos (párr. 169). Así, el discurso de la conservación ambiental es visto por la Corte como una justificación válida para la restricción de derechos y, al mismo tiempo, como una posible herramienta para su ilegítima afectación, dando cuenta del momento de tensión que planteamos.

En este marco, la naturaleza comienza a adquirir un valor propio en las reflexiones de la Corte, aunque no de forma expresa. Por un lado, en el caso pueblos indígenas Kuna de Madungandi y Emberá de Bayano Vs. Panamá, de $2014^{18}$, la Corte dicta su primera sentencia sobre vulneración del derecho a la tutela judicial efectiva por incumplimiento del plazo razonable en procesos penales seguidos por delitos contra el ambiente, en específico, por la tala ilegal de árboles (párr. 185). La tutela judicial efectiva se aplica, así, por primera vez, sobre casos en los que no se estaban tutelando bienes jurídicos humanos. Por otro lado, la naturaleza comienza a estar presente también en materia de

\footnotetext{
${ }^{17}$ Corte IDH. Sentencia del caso de la comunidad indigena Xákmok Kásek vs. Paraguay, de 24 de agosto de 2010.

${ }^{18}$ Corte IDH. Sentencia del caso pueblos indígenas Kuna de Madungandí y Emberá de Bayano y sus miembros Vs. Panamá, de 14 de octubre de 2014.
} 
reparaciones las dos sentencias que se detallan a continuación son ejemplo de aquello: 1) en el caso Sarayaku Vs. Ecuador, de $2012^{19}$, la Corte dictó las primeras medidas de reparación que favorecen a la naturaleza, entre ellas, plantar especies locales de árboles (párr. 294), y 2) en el caso Garífuna de punta piedra y sus miembros Vs. Honduras, de $2015^{20}$, dispuso restaurar las áreas deforestadas, para lo cual, entre otras cuestiones, ordenó la creación de un fondo que incluya como uno de sus objetivos, ahora sí, la recuperación ambiental (párrs. 333-335). Es de destacar que, como se expuso en el apartado anterior, la Comisión y las víctimas ya habían solicitado medidas de reparación similares en casos previos, sin que aquellas peticiones hubieran sido siquiera tratadas por la Corte.

En este contexto se dicta la sentencia que, en mi consideración, representa el punto más alto de la tensión ambiente-derechos humanos, el caso Kaliña y Lokono vs. Surinam, de 2015. Aquí se demandó, entre otras, el establecimiento y continuidad de tres reservas naturales ubicadas en parte del territorio ancestral de dichos pueblos. En este artículo me concentraré en las alegaciones relativas a dos de ellas, las reservas Wia Wia y Galibi. Estas reservas tienen como objetivo la protección de sitios de anidación de tortugas marinas (párr. 81). Los pueblos Kaliña y Lokono tienen, como práctica tradicional precisamente, la recolección de huevos de tortuga marina para su consumo personal. Además, durante varios años se dedicaron a la comercialización de estos huevos, actividad que no es descrita como tradicional (párrs. 74-79). Así, la disputa entre la preservación del ambiente y los derechos de estos pueblos estaba planteada. Esta es, de hecho, reconocida por la Corte en el párrafo 191 de la sentencia al indicar que, si bien a la luz de los estándares internacionales es compatible el control, acceso y participación los pueblos indígenas y tribales en áreas del territorio de una reserva, "resulta razonable que el Estado pueda tener control, acceso y manejo de áreas de interés general, estratégico y de seguridad".

La cita es de especial relevancia, pues lleva a la Corte a considerar que, en el presente caso, no se configuró la violación por la falta de control y manejo exclusivo de las reservas por parte de dichos pueblos, sino tan solo por la falta de mecanismos expresos que garanticen su acceso, uso y participación efectiva en la conservación y aprovechamiento de los beneficios de las mismas (párr. 197). Es decir, por primera vez en su jurisprudencia, no se establece la vulneración del derecho al control exclusivo sobre su propiedad comunal, sino únicamente a la participación en la toma de decisiones sobre la administración de las reservas naturales. El control y, por ende, la propiedad de esas tierras sigue siendo del Estado (párr. 286). De esta forma, se adelanta un criterio

${ }^{19}$ Corte IDH. Sentencia del caso pueblo indigena Kichwa de Sarayaku Vs. Ecuador, de 27 de junio de 2012.

${ }^{20}$ Corte IDH. Sentencia del caso comunidad Garifuna de Punta Piedra y sus miembros vs. Honduras, de 8 de octubre de 2015.

Araucaria. Revista Iberoamericana de Filosofí, Politica, Humanidades y Relaciones Internacionales, año $23, \mathrm{n}^{\circ} 46$. Primer cuatrimestre de 2021. Pp. 505-527. ISSN 1575-6823 e-ISSN 2340-2199 https://dx.doi.org/10.12795/araucaria.2021.i46.25 
inédito hasta el momento respecto del derecho a la propiedad comunal, el de que una vulneración al derecho al territorio (a los recursos naturales) no implica en todos los casos una vulneración al derecho a la tierra (a la propiedad). Hasta este caso la vulneración de alguno de estos derechos conllevaba de forma inseparable la vulneración del otro ${ }^{21}$. Esto, que a mi juicio es una de los datos más relevantes de la sentencia, es muy poco visibilizado dentro de su texto. Sin embargo, como veremos en la siguiente etapa, esta idea es retomada, no sin opiniones disidentes, en la última de las sentencias que estudia este artículo.

Este cambio jurisprudencial es inspirado por la consideración, adelantada por la Corte en la primera sentencia citada en este apartado, de que la preservación del ambiente constituye un objetivo legítimo para restricción de derechos. La consolidación de este precedente, motivada por las particularidades del caso, lleva a la Corte a establecer un criterio central, con el que da cuenta de la tensión existente. Esto es, la necesidad de ponderar los derechos en juego, es decir, entre los derechos colectivos de los pueblos Kaliña y Lokono y la protección del medio ambiente como parte del interés general (párr. 168). Este criterio es reafirmado en las reparaciones del caso, al indicar que cualquier restricción futura sobre los derechos de los pueblos indígenas en las reservas naturales deberá cumplir con los requisitos de legalidad, necesidad, proporcionalidad y el logro de un objetivo legítimo (párr. 286).

El ubicar al ambiente como un interés más a ponderar lleva consigo su no instrumentalización. Solo podemos ponderar entre intereses jurídicos o derechos que tienen el mismo valor jerárquico. Aquí ya se presentan los primeros precedentes de lo que, dos años más tarde, reconocería de forma expresa la Corte: la protección de la naturaleza como un interés en sí mismo.

\subsection{Su protección directa de tipo ecocéntrica: la consagración del derecho al medio ambiente sano como derecho autónomo}

En la línea evolutiva que plantea este artículo, esta etapa se caracteriza por la incorporación expresa del enfoque ecocéntrico al interior de las reflexiones de la Corte IDH. Inicia con la Opinión Consultiva 23/17, de 15 de noviembre del $2017^{22}$ (en adelante OC 23/17), sobre medio ambiente y derechos humanos. Si bien los aportes de esta opinión se efectúan, principalmente, desde la ya asentada protección antropocéntrica instrumental del ambiente, como veremos, dos tipos de avances esenciales respecto de este tipo de protección se dan en esta etapa: 1) la revisión de los estándares establecidos por la Corte sobre la

\footnotetext{
${ }^{21}$ Al respecto se puede ver: (Wences y Russo, 2016: 289).

22 Corte IDH. Opinión Consultiva OC-23/17, medio ambiente y derechos humanos, de 15 de noviembre de 2017.
} 
protección indirecta, y 2) El reconocimiento del derecho ecocéntrico a un medio ambiente sano como un derecho autónomo.

\subsection{1. la revisión de los estándares establecidos por la Corte sobre la protección indirecta}

Sobre los avances de la protección indirecta, siendo muy sintético identifico cuatro: la responsabilidad internacional de los Estados por daños transfronterizos propios y de terceros, los avances en las obligaciones derivadas de los derechos procedimentales y, finalmente, la inclusión del principio de prevención y, en especial, el de precaución.

En primer lugar, la Corte, interpretando el artículo 1.1 de la Convención, determinó que frente a daños ambientales transfronterizos la responsabilidad corresponde al Estado en cuyo territorio o bajo cuya jurisdicción se realizan las actividades contaminantes, pues al ser este el que tiene el control efectivo sobre las mismas, es quien está en posición de impedir que se cause el daño (párrs. 102-104). Este precedente es especialmente relevante, pues llena el vacío jurisdiccional existente a nivel regional sobre daños ambientales transfronterizos. En segundo lugar, la Corte recoge dos avances que, sin ser parte de la respuesta directa dada en la Opinión Consultiva, siguiendo la forma en la que se han venido dando los desarrollos jurisprudenciales en la historia de la Corte, pueden ser interpretados como cartas de intención a futuro: 1) la responsabilidad directa de las empresas en materia de derechos humanos (párr. 155), y 2) la responsabilidad estatal por las actividades de empresas transnacionales registradas en su territorio pero que desarrollan actividades fuera del mismo (párr. 151).

Sobre las obligaciones de procedimiento, en esta etapa se registran dos avances. En primer lugar, se incorpora la transparencia activa en materia ambiental como parte del derecho al acceso a la información (párrs. 221 y 223). Esta obligación que, como vimos, no se contempló en la etapa de protección indirecta antropocéntrica, lleva consigo la idea de que la protección ambiental no es un asunto cuyo debate deba ser abierto únicamente bajo demanda, es decir, bajo petición de una persona o grupo de personas interesadas (transparencia pasiva), sino que, como ya lo venía exponiendo la Corte desde la etapa de tensión, constituye un tema de interés general.

En segundo lugar, la Corte establece que los Estados deben garantizar que los individuos tengan acceso a recursos para impugnar cualquier norma, decisión, acto u omisión que pueda contravenir las obligaciones de derecho ambiental. En la etapa anterior se había declarado la vulneración del derecho al debido proceso por el incumplimiento del plazo razonable dentro de un proceso por daños al ambiente ya existente y regulado en la legislación interna, aquí se da un paso adicional, estableciéndose que en aquellos países 
en los que no exista un proceso judicial para la defensa de las obligaciones en materia ambiental, este debe ser regulado con el fin de garantizar el derecho a la protección judicial (art. 25). Este precedente, de la mano de la declaración del derecho al medio ambiente sano como derecho autónomo, nos puede dirigir a una conclusión de gran relevancia para la protección ecocéntrica de la naturaleza: las obligaciones derivadas del artículo 25 de la Convención harían necesaria la regulación de un recurso judicial efectivo para la garantía de ese interés en sí mismo que representa la naturaleza, es decir, para la garantía de los derechos de la naturaleza.

Finalmente, como parte de los avances de la protección antropocéntrica, se incorporan los principios de prevención y precaución, ambos provenientes del derecho ambiental. En primer lugar, el principio de prevención actúa frente a actividades que causen daños significativos al medio ambiente. La idea del daño significativo, en el marco de este principio, es claramente antropocéntrica, pues, como lo cita la Corte, este se refiere al "daño ambiental en la persona, no el riesgo existente para el medio ambiente o el nivel de degradación ambiental" (párrs. 139140). Está dirigido a salvaguardar una situación legal humana existente, por lo que no es estrictamente preventivo en materia ambiental (Leroy, 2006: 68). Por el contrario, el principio de precaución se aplica de antemano y cumple una función programática. Actúa en casos donde no existe certeza científica sobre el impacto que pueda tener una actividad en el medio ambiente (OC 23/17, párr. 175), es decir, se articula como una regla interpretativa por la cual, ante la duda, se debe favorecer la protección de la naturaleza (OC 23/17, párr. 180). Sin embargo, no cualquier posible daño habilita la aplicabilidad de este principio; el daño debe ser "grave o irreversible", esto es, requiere de un nivel mayor que el estándar aplicable a la obligación de prevención (OC 23/17, nota 426). Así, si bien su alcance es más amplio, su aplicabilidad resulta mucho más compleja, buscando, con ello, y en mi consideración, que el argumento a la protección del ambiente no impida, de forma desmedida, el desarrollo de los procesos productivos humanos. De ahí que las medidas "necesarias" que deben adoptar los Estados en el marco de este principio sean aquellas "eficaces en función de los costos" (OC 23/17, nota. 425), volviendo, una vez más, al análisis de costobeneficio propio de las reflexiones antropocéntricas.

\subsubsection{El reconocimiento ecocéntrico del derecho a un medio ambiente sano como un derecho autónomo}

Siguiendo la línea jurisprudencial sobre la justiciabilidad directa de los derechos económicos, sociales, culturales y ambientales, en la OC 23/17 se consagra, por primera vez, el derecho al medio ambiente sano como un derecho autónomo dentro del Sistema Interamericano. Así lo establece la Corte en el párrafo 55 de la Opinión Consultiva, al indicar que, dada la estrecha conexión 
entre la protección del medio ambiente con el desarrollo sostenible y los derechos humanos, actualmente diversos sistemas de protección de derechos humanos y, en particular, el Sistema Interamericano, reconocen el derecho al medio ambiente sano como un derecho en sí mismo. Este, como menciona expresamente en el párrafo 63 , "es distinto al contenido ambiental que surge de la protección de otros derechos, tales como el derecho a la vida o el derecho a la integridad personal".

De esta forma, la Corte desmarca el derecho autónomo al medio ambiente sano del tipo de protección instrumental o por conexión. Pero no solo aquello, en el párrafo 62 de la Opinión Consultiva desarrolla el contenido de este derecho declarando que, "a diferencia de otros derechos, este protege los componentes del medio ambiente tales como bosques, ríos, mares y otros como intereses jurídicos en sí mismos, aun en ausencia de certeza o evidencia sobre el riesgo a las personas individuales". Con ello, me parece, desmarca este derecho además del tipo de protección antropocéntrica, pues busca:

proteger la naturaleza y el medio ambiente no solamente por su conexidad con una utilidad para el ser humano o por los efectos que su degradación podría causar en otros derechos de las personas, como la salud, la vida o la integridad personal, sino por su importancia para los demás organismos vivos con quienes se comparte el planeta, también merecedores de protección en sí mismos (OC 23/17, párr. 62).

Del citado párrafo se pueden obtener ciertos rasgos que nos permiten dar cuenta de cómo esta construcción se aleja de la titularidad antropocéntrica individual o colectiva del derecho al ambiente. Se podría creer, por ejemplo, que la consagración del derecho a un medio ambiente sano como un derecho autónomo es un mecanismo para evitar un proceso de degradación ambiental que ponga en riesgo a toda la humanidad (dimensión humano-colectiva). Sin embargo, la Corte va un paso más allá al definir este derecho, pues en este caso pone el acento en la protección que ofrece para los demás organismos no humanos. Así, el objetivo no es ya conservar la humanidad, sino a todos los organismos que conviven con ella. Reconoce un nuevo bien jurídico y, con ello, un nuevo sujeto de protección dentro del sistema interamericano, la naturaleza en sí misma. El núcleo de protección que crea la Corte alrededor de este nuevo derecho reconoce derechos para todo el entorno, incluido entes inertes (bosques, ríos, mares) a los cuales identifica como "componentes" del ambiente, incorporando con esto último el enfoque holístico que caracteriza al ecocentrismo. 
En este marco, el 6 de febrero de 2020, en la sentencia del caso Lhaka Honhat Vs. Argentina ${ }^{23}$, se declara, por primera vez dentro de un caso contencioso, la vulneración del derecho al ambiente sano como derecho autónomo. Esta sentencia constituía una oportunidad para llenar el vacío dejado en la OC 23/17 sobre las garantías que se derivan de dicho derecho; sin embargo, como veremos, a pesar de haberse declarado su vulneración la argumentación de la Corte es poco clara y en nada difiere de la ya establecida para la vertiente instrumental-antropocéntrica. En el caso, entre otros, se demandó la vulneración del derecho al medio ambiente sano, en razón del proceso de degradación ambiental producido por el sobrepastoreo de ganado bovino, tala ilegal de bosques e instalación de alambrados por parte de las familias criollas (párrs. 186-189).

Para dar respuesta a las alegaciones presentadas, la sentencia divide el derecho a la tierra de los pueblos indígenas, sobre el cual se declara su vulneración en relación al artículo 21 de la Convención (derecho a la propiedad), del derecho al territorio, el cual lo vincula al artículo 26 del mismo instrumento y, por esta vía, a los derechos al ambiente sano, agua, alimentación e identidad cultural. Esta división, sin lugar a dudas, constituyó un tema de gran controversia al momento de la deliberación. Basta ver la apretada mayoría con la que la se declara vulneración del artículo 26 de la Convención (tres jueces a favor, entre los que se encuentra el voto de calidad de la presidenta y tres en contra), pero, además, y de especial relevancia, el debate que mantienen al respecto los jueces Humberto Sierra Porto (en contra) y Eduardo Ferrer Mac-Gregor (a favor), en sus votos particulares parcialmente disidente y concurrente, respectivamente.

Para el juez Sierra Porto, una protección del territorio por fuera del derecho a la propiedad comunal, "no sólo es jurídicamente incorrecta, sino que se aparta de fundamentos antropológicos y sociológicos esenciales que describen y fundamentan la particularidad de las poblaciones indígenas y tribales "24. Por el contrario, para el juez Ferrer Mac-Gregor este precedente constituye un avance trascendental para el sistema interamericano, pues permite el análisis pormenorizado de todos y cada uno de los elementos que forman parte de los derechos de los pueblos indígenas ${ }^{25}$.

El debate planteado por ambos jueces es de especial complejidad e importancia para el futuro de la protección de los derechos de los pueblos indígenas en el Sistema Interamericano. No pretendo abarcar aquí las aristas que este tema presenta; ello requiere de un análisis que excede las páginas del

\footnotetext{
${ }^{23}$ Corte IDH (2020). Sentencia del caso comunidades indígenas miembros de la Asociación Lhaka Honhat (nuestra tierra) Vs. Argentina, de 6 de febrero de 2020.

${ }_{24}$ Sierra Porto, H. (2020). Voto parcialmente disidente, caso Lhaka Honhat Vs. Argentina, de 6 de febrero de 2020, párr. 18 .

${ }^{25}$ Ferrer Mac-Gregor E. (2020). Voto concurrente, caso Lhaka Honhat Vs. Argentina, de 6 de febrero de 2020, párrs. 12 y 34.
} 
presente trabajo. Sin embargo, se debe llamar la atención sobre el hecho de que, aunque en los votos particulares se presenta a la separación entre el derecho a la tierra y el derecho al territorio como una novedad (positiva o negativa), este precedente ya había sido establecido cinco años antes. Como se mencionó, en el caso Kaliña y Lokono Vs. Surinam, de 2015, se negó la vulneración del derecho a la propiedad comunal (a la tierra) en razón del control exclusivo por parte del Estado de reservas naturales ubicadas en su territorio, al mismo tiempo que, garantizando el derecho a la identidad cultural, circulación y participación política, se reconoció y declaró vulnerado el derecho de acceso a los recursos naturales (al territorio). De esta forma, la división ya había sido establecida; sin embargo, la forma difusa en la que se plantean los intereses en juego en la etapa que clasifico como de tensión, hicieron que el conflicto pasara desapercibido; es más, la sentencia del caso Kaliña y Lokono contó con un voto concurrente conjunto, precisamente, de los jueces Sierra Porto y Ferrer Mac-Gregor, ahora en polos opuestos sobre este mismo punto.

Lo anterior me permite concluir que la mayor visibilidad dada en esta última etapa a las posturas en juego permite, además, ampliar el debate sobre los intereses en conflicto, incorporando cuestiones y tensiones que antes se presentaban ocultas. Esto, en mi consideración, es un efecto positivo de la expresa incorporación del ecocentrismo en esta etapa, pues demanda de los jueces y juezas de la Corte una mayor calidad argumental en el análisis casuístico y, por esta vía, de criterios más precisos para su resolución.

En esta misma línea, estándares más claros se deben crear respecto al enfoque ecocéntrico del derecho al medio ambiente sano como derecho autónomo. Si bien en el caso Lhaka Honhat la Corte reitera el contenido que dota de un enfoque ecocéntrico a este derecho (párr. 207), no es del todo clara al momento de detallar las obligaciones derivadas del mismo. En el párrafo 207 de la sentencia, la Corte establece que rige respecto a la vertiente autónoma de este derecho, citando lo dicho en la OC 23/17 sobre la protección indirecta antropocéntrica del mismo, no solo la obligación de respeto, sino también la obligación de garantía prevista en el artículo 1.1 de la Convención. Dos interpretaciones podemos sacar de lo anterior: o la Corte asimila la vertiente procedimental de este derecho a su vertiente autónoma, en cuyo caso la segunda carecería de garantías propias y, por tanto, de autonomía; o la Corte pretende aplicar las garantías ya establecidas sobre la vertiente procedimental de este derecho, la cual protege al ser humano, a la nueva vertiente autónoma. En este segundo escenario, dichas garantías protegerían ya no a los seres humanos, sino a los componentes de la naturaleza como intereses en sí mismos.

Si buscamos dar coherencia al desarrollo jurisprudencial de la Corte, la segunda sería la interpretación más adecuada, sin embargo, debo reiterar que la Corte no es clara al respecto. El análisis de la interdependencia de 
los derechos realizado para la resolución del caso Lhaka Honhat, si bien se aproxima de forma adecuada a la relación entre los intereses en juego, deja sin desarrollar las obligaciones que habrían sido vulneradas respecto del sujeto específico de protección del derecho al medio ambiente sano como derecho autónomo, la naturaleza (párrs. 243 a 254 y 272 a 289). De ahí que, aunque en los párrafos 280 y 281 de la sentencia la Corte señale las formas en que la explotación ganadera y forestal habían degradado el medio natural, esta no llega a considerar a la naturaleza como víctima dentro del caso (párr. 309). Aquello, en mi consideración, constituye un error. Si lo que dota de carácter autónomo al medio ambiente sano, conforme lo ha expresado la Corte, es su protección dirigida a la naturaleza como poseedora de valores propios, con independencia del ser humano, declarar su vulneración poniendo como víctima exclusiva al ser humano puede, ahora sí, hacer que la protección directa del ambiente carezca de sentido.

\section{Conclusiones}

El análisis jurisprudencial realizado ha dado cuenta de que un enfoque ecocéntrico está surgiendo dentro del Sistema Interamericano y que este, contrario a lo que se podría creer, no es producto de voluntades particulares, sino del cada vez mayor peso que ha ido adquiriendo el ambiente en nuestras reflexiones sobre los derechos humanos. Así visto, el momento ecocéntrico, en línea con lo pronosticado por Leopold, surge no solo como una posibilidad evolutiva, sino como una necesidad ecológica (Leopold, 2017: 181, 182). En este escenario, desconocer su presencia, como vimos en el momento de tensión, resulta no solo un contrasentido, sino, además, una forma de evadir los verdaderos intereses en juego, disminuyendo el debate y, con ello, la calidad argumental esperable para la resolución de temas de esta complejidad.

Resulta fundamental, por tanto, comenzar a teorizar sobre las tensiones existentes entre los intereses antropocéntricos y ecocéntricos presentes actualmente en la Corte IDH, pues solo de esta forma podremos elaborar herramientas adecuadas para su ponderación. La tensión, en este caso, se presenta como virtuosa, como una puerta para llegar a compromisos en la conservación y el desarrollo que den respuestas más precisas a los casos planteados.

La Corte tiene un gran reto por delante, estándares más precisos sobre la protección ecocéntrica del ambiente deben surgir de la mano de la consagración de la naturaleza como víctima dentro del Sistema Interamericano. Quiero resaltar este último punto, pues si algún objetivo propio tiene el nuevo derecho autónomo al ambiente sano creado por la Corte es este, el cambio 
sustancial en la idea de dignidad humana y, por esta vía, de los sujetos capaces de activar el Sistema Interamericano de Derechos Humanos como víctimas. Si, como expresa, este derecho protege a los bosques, ríos, mares y otros, como intereses jurídicos en sí mismos, sus reflexiones deben trascender el argumento de conexión. 


\section{Bibliografía:}

Costa, C. (2009). ¿Ética ecológica o medioambiental? Acta Amazonica, 39(1), pp. 113-120.

Delgado Rojas, J. I. (2018). Dignidad humana = Human dignity. EUNOMÍA. Revista en Cultura de la Legalidad, 15, pp. 176-197.

(2020). Kant y la dignidad humana en la Corte Interamericana de Derechos Humanos. Revista Derechos y Libertades, pp. 241-271.

Descartes, R. (2002 [1637]). Discurso del método; Meditaciones metafísicas. Tecnos.

Dobson, A. (2005). Ciudadanía ecológica. Isegoría, 0(32), pp. 47-62.

Gros Espiell, H. (1991). La Convención Americana y la Convención Europea de Derechos Humanos. Análisis comparativo. Santiago de Chile: Editorial Jurídica de Chile.

Gudynas, E. (2010). La senda biocéntrica: Valores intrínsecos, derechos de la naturaleza y justicia ecológica. Tabula Rasa: revista de humanidades, núm. 13, pp. 45-71.

Gudynas, E. (2011). Los derechos de la Naturaleza en serio: Respuestas y aportes desde la ecología política. En La Naturaleza con Derechos. De la filosofía a la política (Acosta, A. y Martínez, E. compiladores, pp. 239258). Quito: AbyaYala.

Jonas, H. (2015). El principio de responsabilidad: Ensayo de una ética para la civilización tecnológica. Barcelona: Herder.

Kant, I. (1876 [1790]). Crítica del juicio. Seguida de las observaciones sobre el sentimiento de lo bello y lo sublime. Madrid: Librería de Iravedra.

Kant, I. (1988 [1775-1781]). Lecciones de ética. Barcelona: Crítica.

Klett Lasso de la Vega, P., \& Martínez Anguita, P. (2013). Justicia con la Naturaleza. Madrid: Dykinson.

Leff, E. (1995). ¿De quién es la naturaleza? Sobre la reapropiación social de los recursos naturales. Gaceta Ecológica, 37, pp. 28-35.

Leopold, A. (2017). Una ética de la tierra. Madrid: Los Libros de la Catarata.

Leroy, S. F. (2006). Can the Human Rights Bodies be Used to Produce Interim Measures to Protect Environment-Related Human Rights? Review of European Community and International Environmental Law, 15(1), pp. 66-81.

Lovelock, J. (1979). A New Look at Life on Earth. Oxford: Oxford University Press.

Máiz, R. (2011). Igualdad, sustentabilidad y ciudadanía ecológica. Foro Interno, 11, pp. 13-43.

Montalván Zambrano, D. (2020). Justicia ecológica $=$ Ecological justice. EUNOMÍA. Revista en Cultura de la Legalidad, 18, pp. 179-198. 
Shelton, D. (2010). Derechos ambientales y obligaciones en el sistema interamericano de derechos humanos. Anuario de Derechos Humanos, 6, pp.111-127.

Trejos Fernández, J. (1978). Discurso pronunciado en la sesión inaugural de la Conferencia Interamericana Especializada sobre Derechos Humanos celebrada el viernes 7 de noviembre de 1969. En Actas y Documentos de la Conferencia Especializada Interamericana Sobre Derechos Humanos (pp. 407-412), Secretaría General de la OEA.

Wences, I. \& Russo, A. M. (2016). De los derechos de los "miembros de las comunidades" a los derechos de la "comunidad y sus miembros". En La América de los Derechos (Wences, I. y Santolaya, M. coords. pp. 281326). Madrid: Centro de Estudios Políticos y Constitucionales. 
